\title{
Low temperature acoustic properties of amorphous silica and the Tunneling Model
}

\author{
EunJoo Thompson, G. Lawes, J. M. Parpia, R. O. Pohl* \\ Laboratory of Atomic and Solid State Physics, Cornell University, Ithaca, New York 14853-2501
}

(May 25, 2022)

\begin{abstract}
Internal friction and speed of sound of $a-\mathrm{SiO}_{2}$ was measured above $6 \mathrm{mK}$ using a torsional oscillator at $90 \mathrm{kHz}$, controlling for thermal decoupling, non-linear effects, and clamping losses. Strain amplitudes $\epsilon_{\mathrm{A}}=10^{-8}$ mark the transition between the linear and non-linear regime. In the linear regime, excellent agreement with the Tunneling Model was observed for both the internal friction and speed of sound, with a cut-off energy of $\Delta_{o, \min } / \mathrm{k}_{\mathrm{B}}=6.6 \mathrm{mK}$. In the non-linear regime, two different behaviors were observed. Above $10 \mathrm{mK}$ the behavior was typical for non-linear harmonic oscillators, while below $10 \mathrm{mK}$ a different behavior was found. Its origin is not understood.
\end{abstract}

PACS numbers: 61.43.Fs, 62.54.+k, 63.50.+x

The low temperature acoustic, thermal, and dielectric properties of amorphous solids have long been successfully described by the phenomenological Tunneling Model (TM). In this model, the low energy localized vibrational excitations, a common feature of amorphous solids, are described by non-interacting two-level defects which are thought to be caused by tunneling of atoms or groups of atoms between nearly degenerate potential minima. The excitation energy between the two lowest states of the double well potential, $\mathrm{E}=\sqrt{\Delta^{2}+\Delta_{\mathrm{o}}^{2}}$, is determined by the asymmetry, $\Delta$ and the tunneling splitting, $\Delta_{\mathrm{o}}$ [1]. Low temperature internal friction and speed of sound measurements on $a-\mathrm{SiO}_{2}$ using the torsional oscillator technique between 66 and $160 \mathrm{kHz}$ above $50 \mathrm{mK}$ have shown excellent agreement with the TM [2,3]. However, several acoustic and dielectric experiments have indicated deviations from this model below $100 \mathrm{mK}$ [4 [8] and have been interpreted as evidence for tunneling defect interactions [9,10]. This discrepancy provided the impetus for the present study in which we extended the acoustic measurements to $6 \mathrm{mK}$.

In low temperature acoustic measurements, a major cause of uncertainty are thermal decoupling caused by spurious heat input and self heating, non-linear effects resulting from moderate strain amplitudes, and lack of knowledge of the influence of mounting [2,5, 6, 6, 8, 10]. Taking particular care to control these problems, we report here measurements in the extended temperature range which are in excellent agreement with the predictions of the TM with a low energy cut-off in the tunneling state spectrum, $\Delta_{\mathrm{o}, \min } / \mathrm{k}_{\mathrm{B}}=6.6 \mathrm{mK}$. These results emphasize the extreme care with which low temperature acoustic work must be carried out.

The amorphous silica sample, Suprasil - W, $(<5$ ppm $\mathrm{OH}^{-}$impurities, $4 \mathrm{~mm}$ diameter, $22.33 \mathrm{~mm}$ long) was mounted in a torsional composite oscillator resonating at $\sim 90 \mathrm{kHz}$ [11] in a dilution refrigerator $(0.07-2 \mathrm{~K})$ and in a vibrationally isolated dilution refrigerator with a demagnetization stage $(0.006-0.100 \mathrm{~K})$ 12]. In the latter, the sample was surrounded by a $\mathrm{Nb}$ tube $(5.4 \mathrm{~mm}$ i.d.,
$60 \mathrm{~mm}$ long) in order to shield it from residual magnetic fields except for the earth's field $(<0.5 \mathrm{G})$.

Before presenting our results on the acoustic properties, we describe the thermal and strain studies that are crucial for avoiding experimental errors. We begin by considering thermal decoupling of the sample. The temperature of the sample was measured directly by epoxying a $1 \mathrm{k} \Omega \mathrm{RuO}_{2}$ Dale resistor onto the free end of the undriven $a-\mathrm{SiO}_{2}$ sample, with the leads ( $76 \mu \mathrm{m}$ diameter Evanohm) thermally anchored along its length (see Fig. 1 inset). A twin Dale resistor was attached to the base. $\mathrm{RuO}_{2}$ thick film resistors have been successfully used as thermometers from 0.015 to $80 \mathrm{~K}$ in magnetic fields up to $20 \mathrm{~T}$ [13]. The resistances for the two resistors, read with a self-balancing resistance bridge (Linear Research, LR700) using a power $=10^{-15} \mathrm{~W}$ and calibrated against a $\mathrm{He}^{3}$ melting curve thermometer, were found to have identical temperature dependencies above $10 \mathrm{mK}$ with very little scatter, see Fig. 1a. Below $10 \mathrm{mK}$, however, irreproducible resistances for both resistors were observed (error bars). The reason for this irreproducibility is not known, nor are we aware of any measurements on these resistors below $15 \mathrm{mK}$. Because of this irreproducibility, thermal decoupling of the undriven sample can be definitely ruled out only above $10 \mathrm{mK}$. Heating of the oscillator through vibrational noise in this cryostat below $10 \mathrm{mK}$ is nonetheless considered to be unlikely. The lowest frequency mode of the sample, $\mathrm{f} \approx 3 \mathrm{kHz}$, is that in which the stiff oscillator bends the thin $\mathrm{Be}: \mathrm{Cu}$ torsion rod (1 $\mathrm{mm}$ diameter, $3 \mathrm{~mm}$ long). Because of its relatively large metallic thermal conductivity, this heat will be easily removed through the base. In addition, a torsional oscillator in the same refrigerator connected by a hollow Be:Cu torsion rod previously showed no signs of thermal decoupling to $1 \mathrm{mK}$ [14].

Heating can also occur when the oscillator is driven. The strain amplitude, $\epsilon_{\mathrm{A}}$, is defined as the maximum angular displacement between the two ends of the silica sample. The electrical measurement of $\epsilon_{\mathrm{A}}$ was calibrated by reflecting a laser beam off the sample and measur- 
ing its deflection with a photodiode 15]. The power loss from mechanical dissipation is $Q^{-1} f_{\mathrm{o}} \frac{1}{2} G V \epsilon_{\mathrm{A}}^{2}$, where $Q^{-1}$ is the internal friction, $f_{\mathrm{o}}$ the resonant frequency, $G$ the shear modulus, and $V$ the volume of the oscillator. The thermal resistance, $\mathrm{R}_{\mathrm{th}}$, of the oscillator was measured by heating the sample thermometer (inset of Fig. 1) with a controlled power input from the LR700. The data are close to the dashed line which was calculated ignoring the thermal resistance of the glass sample (see Fig. 1 caption). The explanation may be that heat enters the glass over its entire surface by way of the resistor leads. $R_{t h}$ was calculated by adding the thermal resistance of the glass to that shown as a dashed line, and was used to determine the upper limit of the temperature rise of the driven oscillator for a measured mechanical power loss. For example, at $10 \mathrm{mK}, \epsilon_{\mathrm{A}}=10^{-8}$ would result in $\Delta \mathrm{T}$ $=40 \mu \mathrm{K}$. It should be noted, however, that $\epsilon_{\mathrm{A}} \sim 10^{-7}$ would result in a temperature rise of $4 \mathrm{mK}$ at $10 \mathrm{mK}$ in our amorphous sample, a significant source of self heating and error.

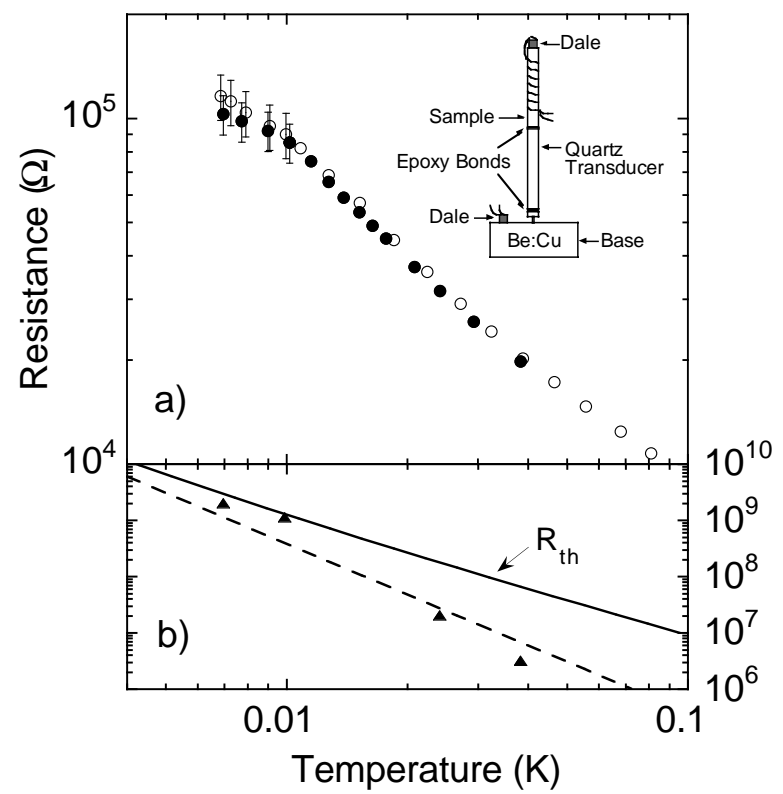

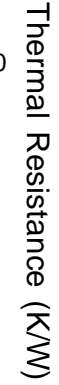

FIG. 1. a) Resistance versus temperature for $1 \mathrm{k} \Omega \mathrm{RuO}_{2}$ Dale thick film resistors mounted on the sample (solid circles) and the base (open circles). The inset shows the experimental setup for this experiment. b) Measured (triangles) and calculated thermal resistances of the torsional oscillator. The solid line is the calculated thermal resistance, $\mathrm{R}_{\mathrm{th}}=\frac{87500}{T^{2}}$ $\left(\mathrm{K}^{3} / \mathrm{W}\right)+\frac{181}{T^{3}}\left(\mathrm{~K}^{4} / \mathrm{W}\right)+\frac{204}{T^{3}}\left(\mathrm{~K}^{4} / \mathrm{W}\right)$, using the method outlined in Ref. [16] and the data table of Ref. [17], where the terms are the thermal resistances of $a-\mathrm{SiO}_{2}$, Casimir, and boundary respectively. The dashed line is the thermal resistance without $a-\mathrm{SiO}_{2}$. (The thermal resistance of the wires connecting the sample to the cryostat was much larger than that of the mounted oscillator.)

The elastic properties of the background were measured by replacing the amorphous sample with a crystal quartz sample of equal size which has negligible internal dissipation. The frequency traces of the background oscillator (quartz on quartz) at different strain amplitudes showed a Lorentzian line shape at all amplitudes and temperatures. Fig. 2a shows two traces taken at nominally $2 \mathrm{mK}$ (i.e. ignoring the $\Delta \mathrm{T}$ due to self-heating). They show that neither the epoxy, nor the Be:Cu metal base lead to non-linear behavior even when the strain amplitude was varied by a factor of 100 . The background internal friction (clamping losses) was found to be slightly temperature dependent while the speed of sound was independent of temperature to within $\sim 0.1 \mathrm{ppm}$, as shown in Fig. 3.

Elastic measurements are sensitive to non-linear effects as can be seen in frequency traces of a driven $a$ $\mathrm{SiO}_{2}$ at increasing driving voltages (peak power dissipation). These traces are plotted in Fig. $2 \mathrm{~b}-\mathrm{d}$ at $27 \mathrm{mK}$, $10.6 \mathrm{mK}$ and $7 \mathrm{mK}$ respectively. The dashed curve is the normalized response of the oscillator carrying a crystal quartz sample. Above $10 \mathrm{mK}$, the lineshapes for $a-\mathrm{SiO}_{2}$ are clearly resolved from that of the quartz sample (background) as seen in Fig. 2b. In contrast to the background, the frequency response of the oscillator with the amorphous sample shows a variety of non-linear behaviors. Above $10 \mathrm{mK}$, with increasing peak strain amplitude, the oscillator exhibits behavior typical for a non-linear harmonic oscillator. (This non-linearity is not seen above $50 \mathrm{mK}$ where it is masked by self-heating [15].) Below $10 \mathrm{mK}$, however, (see Fig. 2c and 2d) the non-linear behavior differs dramatically. With increasing strain amplitudes, the frequency response does not lean to higher frequencies. Instead the response has jumps, plateaus and oscillations. Since the background oscillator shows no such behavior, we conclude that the tunneling entities in $a-\mathrm{SiO}_{2}$ are responsible. This is the first evidence that the tunneling entities themselves may change their behavior below $10 \mathrm{mK}$, although we do not understand the nature of the tunneling entities that can bring about these non-linearities.

The internal friction was only determined when the frequency dependence of the oscillator was clearly in the linear regime, i.e. fit a Lorentzian curve well (solid curves in Fig. 2). In the linear regime, the internal friction was found to be independent of the driving power and $\Delta \mathrm{T}$ to be negligible. At larger strain amplitudes (in the nonlinear regime), the fits yielded higher internal frictions and speeds of sound. As an example, consider Fig 2c; for a peak $\epsilon_{\mathrm{A}}=1.7 \times 10^{-8}$ and a power $=3 \times 10^{-14} \mathrm{~W}$, using a half-width of the non-Lorentzian would lead to an erroneous increase of the internal friction by a factor of 2 and an increase of the speed of sound by $\sim 1 \mathrm{ppm}$. Note that in the most recent investigation of $a-\mathrm{SiO}_{2}$ [8], a double paddle oscillator operating at $\epsilon_{\mathrm{A}} \sim 10^{-7}$ was used, which should lead to considerable non-linearities at low temperatures, at least in our geometry. 

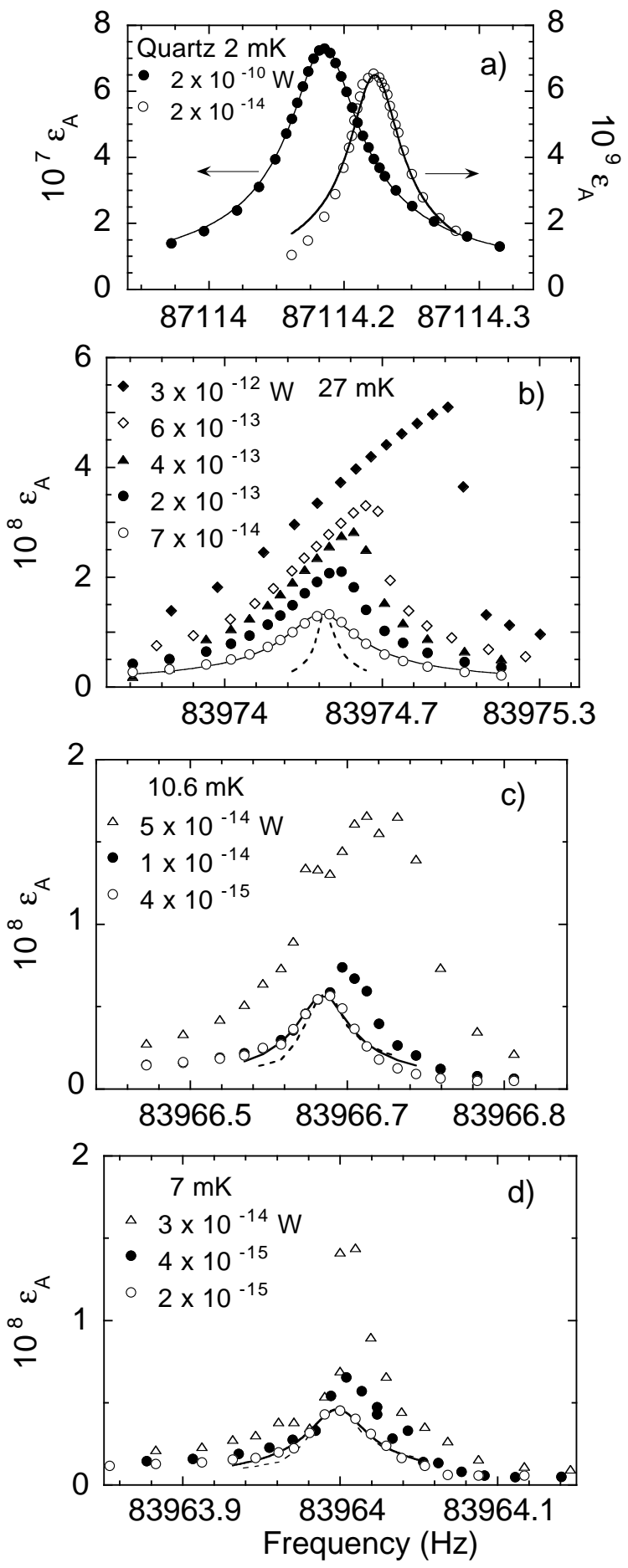

FIG. 2. Frequency response of the background (quartz) and of $a-\mathrm{SiO}_{2}$. a) Lorentzian line shapes of quartz measured at $\sim 2 \mathrm{mK}$ leading to a peak power dissipation of $2 \times 10^{-14} \mathrm{~W}$ and $2 \times 10^{-10} \mathrm{~W}$ are plotted using the right and left $\mathrm{y}$-axes respectively. Solid curves are Lorentzian fits to the data. b-d) frequency response of $a-\mathrm{SiO}_{2}$ at $27 \mathrm{mK}, 10.6 \mathrm{mK}$, and $7 \mathrm{mK}$ for different peak powers. Dashed curves are normalized background Lorentzians at each temperature.

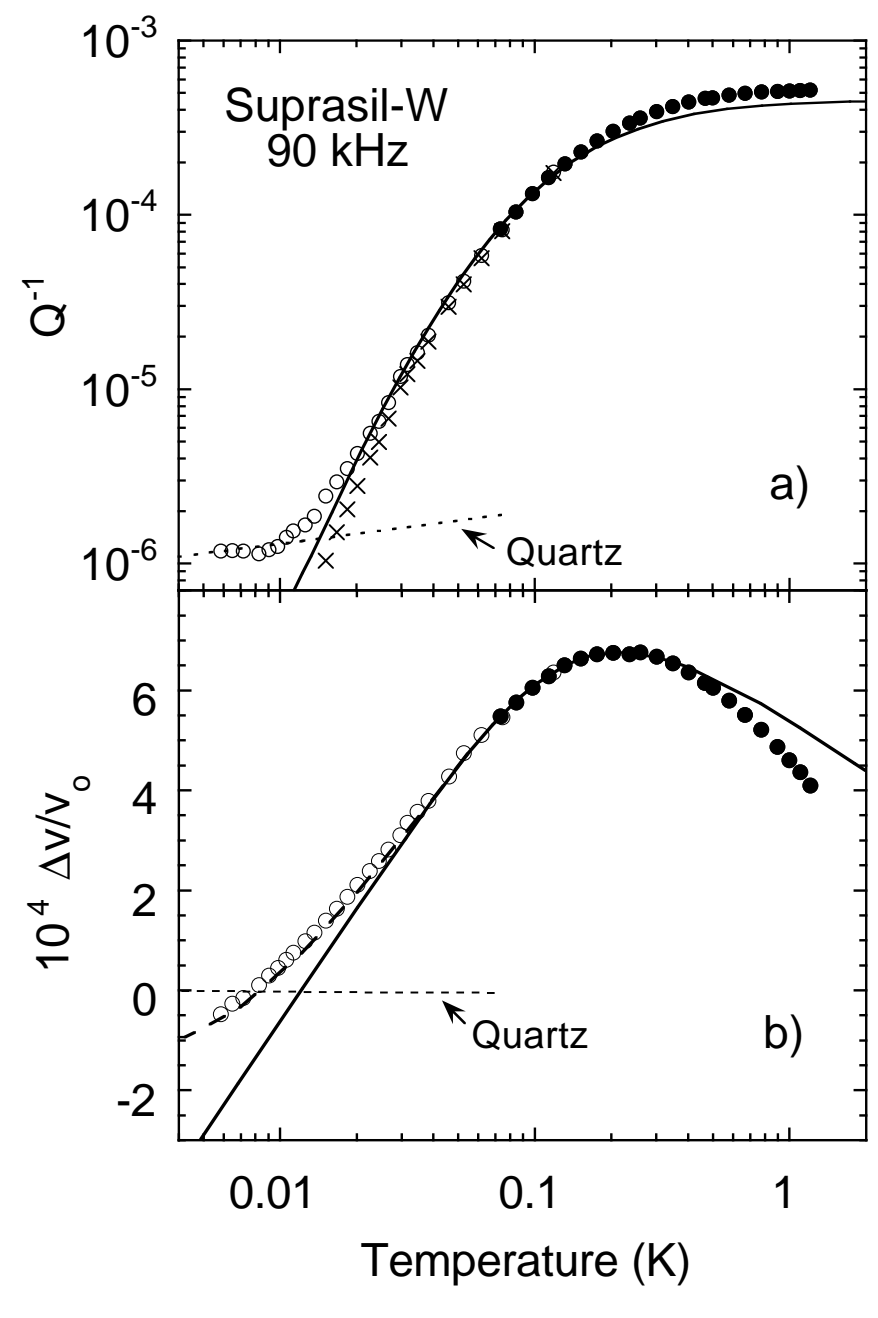

FIG. 3. a) Internal friction and b) relative change of the transverse speed of sound of $a-\mathrm{SiO}_{2}$ (Suprasil-W) at $90 \mathrm{kHz}$ and the background. Solid circles, in dilution cryostat; open circles, in demagnetization cryostat; x's, after subtraction of the background; dashed lines, quartz sample (background); solid curves, TM prediction with parameters from sound velocity measurements; long dash curve, TM prediction with a cut-off energy of $\Delta_{\mathrm{o}, \min } / \mathrm{k}_{\mathrm{B}}=6.6 \mathrm{mK}$.

With the previously identified experimental problems avoided, Fig. 3 shows the internal friction, $\mathrm{Q}^{-1}$, and the relative change of the transverse speed of sound, $\frac{\Delta \mathrm{v}}{\mathrm{v}_{\mathrm{o}}}$ (see eq.1) of $a-\mathrm{SiO}_{2}$ which were determined from the Lorentzian frequency responses, as shown in Fig. 2 as solid curves. Sample heating was always less than $0.5 \%$ of the temperature. The solid and the open circles show the excellent agreement of the data from the two cryostats. Below $10 \mathrm{mK}$, the internal friction approaches a nearly temperature independent value very close to that measured on the quartz sample (dashed line). This close agreement is also evidenced in Figs. 2c and 2d with the frequency responses obtained on both samples (solid and dashed Lorentzians). We conclude that the internal fric- 
tion measured on the $a-\mathrm{SiO}_{2}$ sample below $10 \mathrm{mK}$ is dominated by the background, and use the dashed line to derive the internal friction of the $a-\mathrm{SiO}_{2}$ without this background, shown as x's in Fig. 3a, following the method outlined in Ref [3]. No such correction needs to be applied to the speed of sound, because the background frequency shift is independent of temperature (dashed line in Fig. 3b). The solid curves in Fig. 3 are fits to the TM using a tunneling strength $\mathrm{C}=3.1 \times 10^{-4}$ and a crossover temperature $\mathrm{T}_{\mathrm{co}}=0.08 \mathrm{~K}$, both identical to our previous published values based on the measurements above $50 \mathrm{mK}$ 3. The internal friction shows no deviation from the TM prediction (below $15 \mathrm{mK}$, separating background from the internal friction by the tunneling states would involve large errors). The speed of sound also agrees with the TM prediction between 25 and $500 \mathrm{mK}$. The deviation of $\frac{\Delta v}{v}$ for $\mathrm{T}>500 \mathrm{mK}$ is experimentally well established [9], indicating the existence of channels for defect relaxation other than by single phonon emission. Very remarkable and new, however, is the deviation of $\frac{\Delta v}{v_{0}}$ from the logarithmic temperature dependence below $25 \mathrm{mK}$ which is unambiguous at least to $10 \mathrm{mK}$ (the uncertainty below $10 \mathrm{mK}$ is only due to the thermometer calibration as discussed earlier). This deviation is consistent within the standard TM assuming a cut-off $\Delta_{o, \min } / \mathrm{k}_{\mathrm{B}}$ of the energy distribution of the tunneling states, which affects the speed of sound $\mathrm{v}$ [2]:

$$
\frac{\mathrm{v}(\mathrm{T})-\mathrm{v}_{\mathrm{o}}}{\mathrm{v}_{\mathrm{o}}}=\frac{\Delta \mathrm{v}}{\mathrm{v}_{\mathrm{o}}}=\mathrm{C}\left(\ln \frac{\mathrm{T}}{\mathrm{T}_{\mathrm{o}}}+\frac{\Delta_{\mathrm{o}, \min } / \mathrm{k}_{\mathrm{B}}}{2 \mathrm{~T}}\right),
$$

where $\mathrm{v}_{\mathrm{o}}$ is the speed of sound at some reference temperature $T_{0}$, and $C$ is the tunneling strength. The longdashed curve, calculated with $\Delta_{\mathrm{o}, \min } / \mathrm{k}_{\mathrm{B}}=6.6 \mathrm{mK}$, fits the data in Fig. 3b well. Note that a cut-off should not affect the internal friction significantly, in agreement with the experimental findings. Evidence for a cut-off energy has also been obtained recently from heat pulse measurements of $a-\mathrm{SiO}_{2}\left(\Delta_{\mathrm{o}, \mathrm{min}} / \mathrm{k}_{\mathrm{B}}=3.1 \mathrm{mK}\right)$ [18], and from dielectric measurements on a multi-component alumosilicate glass $(12.2 \mathrm{mK})$ 19]. While such a cut-off may indeed be caused by interaction between the tunneling defects, our data show no evidence for the effects of such interactions beyond this gap.

In conclusion, our measurements have shown no evidence below $500 \mathrm{mK}$ for a deviation from the predictions of the standard Tunneling Model provided we include a cut-off energy in the $\mathrm{mK}$ range. Our results disagree with the earlier studies [5, 6, 8, 10] in which much weaker temperature dependences of both internal friction and speed of sound than predicted by the model had been observed, although at different frequencies $(\leq 14 \mathrm{kHz})$. Those measurements had been interpreted as evidence for defect interactions at temperatures as high as $100 \mathrm{mK}$ [9 10]. We suggest that an alternative explanation may be based, at least in part, on experimental problems common to acoustic experiments such as self-heating and non-linear responses at large strain amplitudes, which can lead to significant errors as we have shown here. It appears essential that all previous evidence for defect interactions be inspected meticulously in order to convincingly exclude these sources of errors. This is not feasible with the published information. Therefore, the fascinating problem of interactions between the tunneling defects should be left as an open question at this time.

We thank Eric Smith and Ch. L. Spiel for their help and many stimulating discussions and Kris Poduska for the loan of the LR700 resistance bridge. We also thank J. Classen for sending us his preprint [8]. This work was supported by the National Science Foundation, grant No.DMR-970972, and DMR9705295.

[1] W. A. Phillips, Rep. Prog. Phys. 50, 1657 (1987).

[2] J. E. Van Cleve, Ph.D. thesis, Cornell University, 1991, unpublished.

[3] K. Topp, E. Thompson, and R. O. Pohl, Phys. Rev. B 60, 898 (1999).

[4] H. Nishiyama, H. Akimoto, Y. Okuda, and H. Ishimoto, J. Low Temp. Phys. 89, 727 (1992).

[5] P. Esquinazi, R. Koenig, and F. Pobell, Z. Physik 87, 305 (1992).

[6] J. Classen et al., Ann. Physik 3, 315 (1994).

[7] S. Rogge, D. Natelson, B. Tigner, and D. Osheroff, Phys. Rev. B 55, 11256 (1997).

[8] J. Classen, T. Burkert, E. Enss, S. Hunklinger, submitted to PRL.

[9] C. Enss and S. Hunklinger, Phys. Rev. Lett. 79, 2831 (1997).

[10] A. Burin, D. Natelson, D.and Osheroff, and Y. Kagan, in Tunneling Systems in Amorphous and Crystalline Solids, edited by P. Esquinazi (Springer Verlag, Berlin, 1998).

[11] D. G. Cahill and J. E. Van Cleve, Rev. Sci. Instr. 60, 2706 (1989).

[12] J. Parpia et al., Rev. Sci. Instr. 56, 437 (1985).

[13] Q. Li et al., Cryogenics 26, 467 (1986).

[14] J. Parpia and T. Rhodes, Phys. Rev. Lett. 51, 805 (1983).

[15] X. Liu, E. Thompson, B. E. White Jr., and R. O. Pohl, Phys. Rev. B 59, 11767 (1999).

[16] E. T. Swartz and R. O. Pohl, Rev. Mod. Phys. 61, 605 (1989).

[17] J. D. N. Cheeke, H. Ettinger, and B. Hebral, Can. J. Phys. 54, 1749 (1976).

[18] P. Strehlow and M. Meißner, Physica B 263, 273 (1999).

[19] P. Strehlow, et. al. submitted to PRL. 